\title{
Twenty-four-Hour Ambulatory Blood Pressure Monitor Heart Rate: A Potential Marker for Gestational Hypertension in at-Risk Women
}

Corenthian J. Booker, M.D., M.M.Ci. ${ }^{1}$, William C. Dodson, M.D. ${ }^{2}$, Allen R. Kunselman, M.A. ${ }^{3}$, John T. Repke, M.D., F.A.C.O.G. ${ }^{2}$, and Richard S. Legro, M.D. ${ }^{2}$

${ }^{1}$ Division of Maternal Fetal Medicine, Department of Obstetrics and Gynecology, University of North Carolina School of Medicine, Chapel Hill, North Carolina

${ }^{2}$ Department of Obstetrics and Gynecology, Penn State University College of Medicine

${ }^{3}$ Division of Biostatistics, Department of Public Health Sciences, Penn State College for Medicine, Hershey, Pennsylvania

\section{Abstract}

We prospectively correlated the 24-hour ambulatory blood pressure measurements (ABPM) to conventional sphygmomanometer blood pressure measurements (CSM) in women at risk for gestational hypertensive disorders (GHTNDs) and identified predictive factors from ABPM for GHTND. We analyzed 73 women with $\geq 1$ risk factor for developing a GHTND. Using both the CSM and ABPM, the systolic blood pressure, diastolic blood pressure, mean arterial pressure (MAP), and heart rate (HR) were measured for 24 hours during three periods (14 to 24 weeks; 24 to 32 weeks; and 33 weeks to delivery). Correlation between the CSM and ABPM lessened as pregnancy progressed. Seventeen (25\%) of women developed a GHTND. MAP variability increased in the GHTND group versus those without a GHTND. The odds of developing a GHTND increased 1.5 times for every 1 beat per minute increase in the ABPM 24-hour HR at visit 1 and reversed by visit 3 . In women at risk for a GHTND, CSM and ABPM correlate less well as pregnancy advances. HR changes in at-risk women may be a marker for the development of a GHTND and may reflect increased sympathetic activity and/or decreased baroreceptor sensitivity.

\section{Keywords}

ambulatory blood pressure monitor; preeclampsia; gestational hypertension and heart rate

Gestational hypertensive disorders (GHTNDs), which include gestational hypertension without proteinuria, preeclampsia (PE), eclampsia, and chronic hypertension, are the second leading cause for maternal mortality in the United States according to the National High Blood Pressure Education Program Working Group Report. ${ }^{1}$ Reliable methods to predict the development of a GHTDS are elusive. ${ }^{2}$ Currently, serial blood pressure (BP) monitoring

Copyright $@ 2012$ by Thieme Medical Publishers, Inc.

Address for correspondence and reprint requests: Corenthian J. Booker, M.D., 101 Manning Drive, Chapel Hill, NC 27514 (cjbooker@med.unc.edu).

Note

The Spacelabs monitors were loaned to us for the period of this study. There is no financial conflict of interest for this study. R.S.L. reports lecture fees from Serono. 
using the conventional sphygmomanometer (CSM) during prenatal visits is the gold standard to assess BP during pregnancy.

Random BP measurements obtained at a single point in time may fail to capture hypertension due to daily fluctuations. Data from nonpregnant patients with essential hypertension suggest that the ambulatory BP monitor (ABPM), which measures BP, repetitively, is superior to CSM in accurately measuring ambient BP. ${ }^{3,4}$ In the nonpregnant population, the ABPM is used to distinguish essential hypertension from "white coat hypertension" to monitor response to drug therapy and to determine risk for future endorgan damage. ${ }^{5}$ The ABPM collects continuous heart rate (HR) and BP measurements at predetermined intervals over a 24 - to 48-hour period. In combination with an activity log, analysis of systolic BP (SBP), diastolic BP (DBP), mean arterial pressure (MAP), pulse pressure (Pp), and HR over 24 to 48 hours provides insight into individual variability over time. Analysis of ABPM measurements to determine BP variability, diurnal variation, and HR variation may be diagnostically superior to the CSM in the evaluation of hypertension. ${ }^{6,7}$ Nevertheless, the ABPM has not been adopted widely for use during pregnancy.

Use of the ABPM in pregnant women as a general screening tool to predict a GHTND or PE is still inconclusive.$^{8-10}$ Current data suggest that the predictive value of the ABPM for GHTNDs ranges from 15 to $85 \% .^{10-15}$ These studies attempted to improve the efficiency of the ABPM positive predictive value by combining the monitor with other measurements such as ultrasound Doppler, as well as ABPM HR monitoring. Kyle et al found an improvement of 31 to $45 \%$ in the positive predictive value by combining HR and MAP compared with MAP alone. ${ }^{11}$ Although validation studies in both low-risk and high-risk populations exist, we saw the greatest utility for the use of an AMBP monitor in an at-risk population. The primary objective of this study was to assess the correlation of ABPM with CSM in women at high risk for developing a GHTND with or without proteinuria and further to characterize the predictive value of ABPM parameters to identify women who develop GHTNDs.

\section{Materials and Methods}

\section{Design, Setting, and Participants}

We performed a prospective, observational study of BP measurement by two methodologies (CSM and ABPM) and enrolled 73 women from the Central Pennsylvania area. The Institutional Review Board at the Penn State Milton S. Hershey Medical Center approved this study. Every woman gave written informed consent to participate in the study. Women were considered eligible if they had one or more risk factors for developing GHTNDs. The risk factors were: (1) chronic hypertension (defined by SBP > $140 \mathrm{~mm} \mathrm{Hg}$ and/or DBP > 90 $\mathrm{mm} \mathrm{Hg}$ prior to 20 weeks' gestation), (2) history of PE, (3) history of gestational hypertension, (4) obesity (body mass index [BMI] $>30 \mathrm{~kg} / \mathrm{m}^{2}$ ), (5) diabetes mellitus, (6) history of gestational diabetes, (7) renal disease, (8) diagnosed thrombophilias, (9) collagen vascular disease, (10) history of abruptio placentae, (11) history of intrauterine growth restriction related hypertension (defined by a history of intrauterine growth restriction with a history of chronic hypertension), (12) strong family history of PE (first-degree relative), (13) history of fetal death, (14) polycystic ovary syndrome, and (15) infertility. Exclusion criteria included multiple pregnancy and patients without a formal obstetric intake exam to identify these risk factors. We excluded a multiple pregnancy because we expected to have too few subjects to analyze. Development of a GHTND was defined to include any of the following diagnoses: gestational hypertension, PE, or HELLP syndrome (hemolysis; elevated liver enzymes; low platelet count) as determined by the health care provider and confirmed by review of the patients health record. The definitions of each diagnosis were defined by the 
Report of the National High Blood Pressure Education Program Working Group on High Blood Pressure in Pregnancy. ${ }^{1}$ All subjects were followed until they were admitted into the hospital or delivered.

\section{Study Procedures}

Women were scheduled for their study visits in the General Clinical Research Center (GCRC) at the Milton S. Hershey Medical Center. The study included three visits to the GCRC, the first at 14 to 24 weeks' gestation, the second at 24 to 32 weeks' gestation, and the third at 32 weeks' gestation to delivery. Each visit included: (1) a height and weight check, (2) three BP measurements using the CSM, and (3) fitting of the ABPM (Spacelabs Healthcare, Issaquah, WA) on the same arm (primarily the nondominant arm) as the CSMbased measurements for a 24-hour measurement period. The ABPM consists of small device connected to the BP cuff by a rubber tube that traverses along the flank to the back of the arm. A waist or shoulder harness supports the device. During each visit, the subject was instructed on troubleshooting the device and was given a diary to record their activity during the BP collection periods.

\section{BP Assessment}

CSM BP Assessment (Office Measurement)—A subject rested in the GCRC in a quiet room for 15 minutes prior to obtaining an auscultatory CSM BP determined by Korotkoff V. The CSM BP was collected in the sitting position on the nondominant arm in triplicate. The ABPM cuff was placed on the nondominant arm if the CSM and ABPM measurements were $<10 \mathrm{~mm} \mathrm{Hg}$ of each other; otherwise, it was placed on the arm with the highest BP measurement. The BP cuff size was selected based on the subject's BMI. The 12 $\times 26 \mathrm{~cm} \mathrm{BP}$ cuff was used for BMIs $<30 \mathrm{~kg} / \mathrm{m}^{2}$; otherwise the $12 \times 40-\mathrm{cm} \mathrm{BP}$ cuff was used.

ABPM Assessment-The Spacelabs ABPM was placed around the bicep and programmed to record SBP, DBP, MAP, HR, and Pp measurements every 30 minutes for 24 hours. The ABPM premeasurement indicator "chimed" prior to the BP and HR measurement during the day and turned off during the night. The women logged their activity, sleep, and wake times in a provided diary.

ABPM BP and Pregnancy Data Evaluation-For each subject and visit, BP measurements obtained by the APBM were reviewed and measurements that showed irregular rest activity or nonphysiological readings (i.e., DBP $>$ SBP), recorded $<14$ wakefulness measurements, or recorded $<7$ sleep measurements were set to missing for analytical purposes. The activity and sleep cycles of the subjects were determined from their diaries. Demographic, delivery, and neonatal information were abstracted from the prenatal and delivery records. We could not determine GHTND status for four subjects because they did not give authorization for the release of their obstetric records.

\section{Statistical Analysis}

We assumed that the null correlation coefficient between the ABPM and CSM methods of measuring BP would be 0.75 and that the study would yield a correlation coefficient 0.90 at any given visit. Furthermore, we assumed $10 \%$ subject dropout for the study. With these assumptions, a sample size of 48 subjects provided at least $90 \%$ power to detect a correlation coefficient of 0.90 between the ABPM and CSM BP measurements using a twosided test and a type I error of 0.05 .

The measurements assessed were SBP, DBP, MAP, HR, and Pp. The three CSM BP measurements obtained at the GCRC were averaged per visit. Similarly, the BP data recorded by the ABPM were averaged per visit for each of the time cycles: 24-hour, 
wakefulness time, and sleep time. The Pearson correlation coefficient $(r)$ and associated 95\% confidence interval (CI) were used to assess the correlation between the averaged APBM and CSM BP measurements at each of the three visits. The partial Pearson correlation coefficient was used to assess the correlation between birth weight and the averaged ABPM measurements at each of the three visits, adjusting for gestational age. Linear mixed-effects models were fit to assess the effect of GHTND status on the ABPM measurements at each visit. The linear mixed-effects model accounts for the within-subject variability inherent in studies with repeated measurements per subject. Logistic regression models were fit to assess the predictive value of ABPM HR measurements on GHTND status. No adjustment for multiple hypothesis testing has been done on secondary outcomes as these were exploratory in nature. All hypothesis tests were two-sided, and all analyses were performed using SAS software, version 9.2 (SAS Institute Inc., Cary, NC). $p$ values less than 0.05 were considered statistically significant.

\section{Results}

\section{Enrollment and Demographics}

Two hundred fifty subjects were screened to participate in the study and 73 enrolled into the study (Fig. 1). Demographic and delivery characteristics for the 73 enrolled subjects overall and by GHTND status are shown in Table 1. Of the 73 subjects, 17 developed GHTNDs (GHTN only: $n=4$; PE only: $n=12$; chronic hypertension only: $n=1$ ). Fifty-two women did not develop GHTND. Of the women who developed GHTNDs, 10 (59\%) had more than one risk factor for developing GHTNDs, whereas $14(27 \%)$ of the women who did not develop a GHTND had more than one risk factor $(p=0.02)$.

\section{Correlation of ABPM Parameters to CSM}

When assessing all enrolled subjects, there was a positive correlation between the 24-hour ABPM and CSM measurements of MAP at all three gestational age visits (Fig. 2). The correlation between the 24-hour ABPM and CSM measurements of MAP weakened beyond the first visit. This same pattern of positive correlations and subsequent weakening of the association as gestational age advanced between ABPM and CSM measurements also were noted for wakefulness and sleep time cycles of MAP as well as for 24-hour, wakefulness, and sleep time measurements of SBP, DBP, and Pp (data not shown).

\section{Correlation of the ABPM and CSM in Subjects without a GHTND}

Among women without a GHTND, CSM and 24-hour ABPM measurements of MAP were positively correlated and strongest in the first and weakest in the second gestational visit (visit 1: $r=0.74,95 \%$ CI: $0.56,0.85$; visit 2: $r=0.39,95 \%$ CI: 0.06, 0.64; and visit $3: r=$ $0.51,95 \%$ CI: $0.20,0.73$ ). Similarly, the correlations between the ABPM and CSM measurements of MAP for the wakefulness and sleep time cycles were strongest in the first and typically weakest in the second gestational visit. This pattern also held when assessing the correlation between 24-hour, wakefulness, and sleep cycle ABPM data with CSM for SBP, DBP, and Pp measurements (data not shown).

\section{BP Variability in Women with GHTNDs versus Women without a GHTND}

The ABPM measurements of SBP, DBP, MAP, and Pp during 24-hour, wakefulness, and sleep time cycles were compared between women with GHTNDs and those without a GHTND. Women with GHTNDs had higher ABPM values compared with subjects without GHTNDs regardless of the BP measurement, time cycle, or gestational visit assessed (24hour ABPM MAP at visit 1 mean difference $=6.0 \mathrm{~mm} \mathrm{Hg}$, 95\% CI: $2.9,9.2, p<0.001 ; 24$ hour ABPM MAP at visit 2 mean difference $=7.7 \mathrm{~mm} \mathrm{Hg}, 95 \%$ CI: 3.4, 11.9, $p<0.001$; 
and 24-hour ABPM MAP at visit 3 mean difference $=9.9 \mathrm{~mm} \mathrm{Hg}, 95 \% \mathrm{CI}: 2.8,17.1, p=$ $0.007)$. Furthermore, we noticed increased 24-hour MAP variability as pregnancy progressed (Fig. 3).

\section{Association of ABPM HR with GHTNDs}

The odds of developing a GHTND increased 1.5 times for every 1 beat per minute increase in the ABPM 24-hour HR at visit 1 (odds ratio $=1.5,95 \%$ CI: 1.0, 2.3, $p=0.05$ ). However, at visit 2 the odds of developing a GHTND were no longer statistically significant (odds ratio $=1.1,95 \%$ CI: $0.9,1.5, p=0.26$ ), and by visit 3 , the odds reversed for every 1 beat per minute increase in the ABPM 24-hour HR (odds ratio $=0.6,95 \%$ CI: $0.3,1.0, p=0.04$ ). The ABPM 24-hour HR profiles for women with GHTNDs and women without GHTNDs at each of the three gestational age visits are shown in Fig. 4.

\section{ABPM and Birth Weight}

Adjusting for gestational age, birth weight, and 24-hour ABPM MAP measurements were negatively correlated at all visits, but the association was relatively weak and not significant (visit 1: $r=-0.14,95 \%$ CI: $-0.39,0.13$; visit $2: r=-0.20,95 \%$ CI: $-0.47,0.11$; and visit $3: r$ $=-0.26,95 \%$ CI: $-0.54,0.07)$. Similar negative correlations held between birth weight and the ABPM measurements of MAP for the wakefulness and sleep time cycles. This pattern also held when assessing the correlation between birth weight and 24-hour, wakefulness, and sleep cycle ABPM data for SBP, DBP, and Pp measurements (data not shown).

\section{Discussion}

In this study, we examined patients at risk for GHTNDs and evaluated correlations between ABPM and CSM measurements. We report a positive correlation between the CSM and ABPM in an at-risk population, which lessens after the first half of pregnancy. In women without GHTNDs, the correlation of ABPM and CSM measurements were positively correlated and strongest in the first and weakest in the second gestational visit. Women with GHTNDs had higher ABPM values compared with subjects without a GHTND, regardless of the BP measurement, time cycle, or gestational visit. We found no significant correlations between 24-hour ABPM measures and birth weight. Finally, the odds of developing a GHTND increased as HR increased early in pregnancy; however, the odds were reversed in late pregnancy. We postulate that this phenomenon occurred because women with GHTNDs have increased sympathetic tone and/or decreased baroreceptor sensitivity presenting as increased HR variation early in pregnancy and increased BP variability later in pregnancy compared with those without a GHTND.

Our findings are consistent with other studies comparing the CSM to ABPM in women who develop a GHTND. Hermida et al reported ${ }^{7}$ an elevated 24-hour ABPM circadian rhythm among women with normal BP, gestational hypertension, and PE. In addition, they showed an increased BP difference between the gestational hypertension group versus the PE group but no difference in the amplitude. They did not report an absent or decreased nocturnal BP dipping effect in their study in either the gestational hypertension group or the PE group. ${ }^{16}$ In addition to their large sample size, their average patient BMI was $27 \mathrm{~kg} / \mathrm{m}^{2}$ whereas ours was $33 \mathrm{~kg} / \mathrm{m}^{2}$. This may suggest that more obese women with a GHTN or PE may have a decreased vascular response compared with thinner women.

The effects of an increased BP variability during pregnancy are uncertain. In the nonobstetric population, a blunted variability is more associated with poor cardiovascular outcomes, but this has not been documented in relation to perinatal morbidity in an obstetric population. There is evidence that there is a difference in the variability and nocturnal 
dipping of women who develop a GHTND, but these findings have not been consistently reproduced. Given the heterogeneity of GHTNDs, it is conceivable that BP variability is more associated with certain types than others. Further investigation is needed to understand how to consistently evaluate BP variability in pregnancy and its role in identifying patients who develop a GHTND.

The etiology of the HR changes and its association in those who develop a GHTND is uncertain and beyond the scope of our study. In normal early pregnancy, the HR increases with a slight increase in response to increased cardiac output in later pregnancy. Studies suggest that HR responses and baroreceptor reflex sensitivity is blunted in pregnancy. Molino et al demonstrated this when evaluating baroreceptor sensitivity among women with normal pregnancies compared with pregnant women diagnosed with PE. ${ }^{17}$ Faber et al evaluated the HR variability and BP variability in women with pregnancy-induced hypertension, chronic hypertension, and PE. They report a unique pattern and relative increases in HR and BP variability among each hypertensive diagnosis compared with the normotensive pregnancy. ${ }^{18}$ In our study, the increased HR with slight increase in BP changes may reflect decreased baroreceptor sensitivity. Also, the early increased BP may be an effect of increased sympathetic tone in those who later develop a GHTND. We postulate that these findings are an average, but increased total averaged effect given our heterogenous mixture of obesity, chronic hypertension, GHTN, and PE in our analysis. It is unclear which subset these findings are most related to. Helmreich et al performed a metaanalysis reporting the effects of obesity and HR changes in pregnancy. They found that obese (BMI $230 \mathrm{~kg} / \mathrm{m}^{3}$ ) women had increased HR compared with women with BMI ranging between 26 and $29 \mathrm{~kg} / \mathrm{m}^{3} .{ }^{19}$

Previous studies support our trend of a rise in BP associated with decreased birth weight, although the effect is small, which may explain why our relatively limited, small sample size failed to detect a significant association. Waugh et al evaluated women with a diagnosis of gestational hypertension and found a concomitant fall of birth weight of $67.5 \mathrm{~g}$ with an increase of $5 \mathrm{~mm} \mathrm{Hg}$ of DBP. ${ }^{20}$ Maggioni et al found a similar inverse relationship between DBP and fetal weight. ${ }^{21}$ In particular, they found an association with hematocrit and circadian amplitude and intrauterine growth restriction in women with pregnancy-induced hypertension. Our study attests to the relationship between DBP and fetal growth rate and that its effects are most apparent in the early to mid third trimester. This information can only be assessed by the nighttime diastolic BP measured by the ABPM and cannot be demonstrated by the CSM.

One of the primary limitations of our study is the sample size. Another is the design, in which those most severely affected with GHTNDs are likely to drop out due to obstetric complications. We had a noncompletion rate of $27 \%$ overall, which resulted from a combination of early delivery as well as study dropout due to noncompliance and elected discontinuation of the study. To evaluate the potential confounding effects of attrition, we did a completer's analysis and noted a similar direction and magnitude of relationships. However, these data suggest that our most valid and generalizable findings stem from our first visit. The recruitment of these women was based on physician referral and reassessment of their risk factors and thus is subject to selection bias. The activity and sleep cycles of the subjects were inferred from their diaries and errors in the true sleep cycle written by the subject and/or their interpretation could have occurred. Given the heterogeneity of these disorders, our reported findings may be influenced more by one disorder than the others. The strengths of our study include the prospective design and the focus on a group of at-risk women for gestational hypertension. Our data set also includes BP and HR information throughout pregnancy, and not just at one time point. Last, our study is generalizable to those caring for populations at risk for a GHTND, and therefore we conclude that the ABPM 
can be considered as a means to assess BP ranges on an outpatient basis in this at-risk group of women.

Our study and previous studies do not support the routine use of ABPM to detect women likely to develop a GHTND. ABPM remains an experimental measure in pregnancy. However, our visit 1 data suggest that employing the ABPM in the early at-risk pregnancy may be the most optimal time to detect physiological changes in those at risk for developing a GHTND. Future direction of research may investigate the clinical utility of HR changes measured by the ABPM in the prediction of specific GHTNDs and in well-defined at-risk groups.

\section{Acknowledgments}

We would like to thank the study coordinators Barb Scheetz and Emily George for their assistance in completing this study. We would also like to thank John Clark with Spacelabs for the generous use of the monitors. This work was supported by a National Perinatal Foundation grant, a PHS GCRC grant MO1 RR 10732, and construction grant C06 RR016499 to Pennsylvania State University. We thank also Urs A. Leuenberger, M.D., and Mark Kozak, M.D., for their insight into potential mechanisms for the heart rate findings.

\section{References}

1. Report of the National High Blood Pressure Education Program Working Group on High Blood Pressure in Pregnancy. Am J Obstet Gynecol. 2000; 183:s1-s22.

2. Barton JR, Sibai BM. Prediction and prevention of recurrent preeclampsia. Obstet Gynecol. 2008; 112(2 Pt 1):359-372. [PubMed: 18669736]

3. Dolan E, Stanton A, Thijs L, et al. Superiority of ambulatory over clinic blood pressure measurement in predicting mortality: the Dublin outcome study. Hypertension. 2005; 46:156-161. [PubMed: 15939805]

4. O'Brien E, Beevers G, Lip GY. ABC of hypertension. Blood pressure measurement. Part IIIautomated sphygmomanometry: ambulatory blood pressure measurement. BMJ. 2001; 322:1110 1114. [PubMed: 11337446]

5. Feldman DM. Blood pressure monitoring during pregnancy. Blood Press Monit. 2001; 6:1-7. [PubMed: 11248754]

6. Montan S, Choolani M, Arulkumaran S, Ratnam SS. Automated 24-hour ambulatory blood pressure monitoring in preeclampsia. J Perinat Med. 1995; 23:353-358. [PubMed: 8606340]

7. Hermida RC, Ayala DE, Iglesias M. Predictable blood pressure variability in healthy and complicated pregnancies. Hypertension. 2001; 38(3 Pt 2):736-741. [PubMed: 11566967]

8. Peek M, Shennan A, Halligan A, Lambert PC, Taylor DJ, De Swiet M. Hypertension in pregnancy: which method of blood pressure measurement is most predictive of outcome? Obstet Gynecol. 1996; 88:1030-1033. [PubMed: 8942848]

9. Biswas A, Choolani MA, Anandakumar C, Arulkumaran S. Ambulatory blood pressure monitoring in pregnancy induced hypertension. Acta Obstet Gynecol Scand. 1997; 76:829-833. [PubMed: 9351407]

10. Bergel E, Carroli G, Althabe F. Ambulatory versus conventional methods for monitoring blood pressure during pregnancy. Cochrane Database Syst Rev. 2002; (2):CD001231. [PubMed: 12076403]

11. Kyle PM, Clark SJ, Buckley D, et al. Second trimester ambulatory blood pressure in nulliparous pregnancy: a useful screening test for pre-eclampsia? Br J Obstet Gynaecol. 1993; 100:914-919. [PubMed: 8217973]

12. Higgins JR, Walshe JJ, Halligan A, O’Brien E, Conroy R, Darling MR. Can 24-hour ambulatory blood pressure measurement predict the development of hypertension in primigravidae? $\mathrm{Br} \mathrm{J}$ Obstet Gynaecol. 1997; 104:356-362. [PubMed: 9091016]

13. Bellomo G, Narducci PL, Rondoni F, et al. Prognostic value of 24-hour blood pressure in pregnancy. JAMA. 1999; 282:1447-1452. [PubMed: 10535435] 
14. Benedetto C, Valensise H, Marozio L, Giarola M, Massobrio M, Romanini C. A two-stage screening test for pregnancy-induced hypertension and preeclampsia. Obstet Gynecol. 1998; 92:1005-1011. [PubMed: 9840567]

15. Hermida RC, Ayala DE, Mojón A, et al. Blood pressure excess for the early identification of gestational hypertension and preeclampsia. Hypertension. 1998; 31:83-89. [PubMed: 9449396]

16. Hermida RC, Ayala DE, Mojón A, et al. Differences in circadian blood pressure variability during gestation between healthy and complicated pregnancies. Am J Hypertens. 2003; 16:200-208. [PubMed: 12620698]

17. Molino P, Veglio F, Genova GC, et al. Baroreflex control of heart rate is impaired in preeclampsia. J Hum Hypertens. 1999; 13:179-183. [PubMed: 10204814]

18. Faber R, Baumert M, Stepan H, Wessel N, Voss A, Walther T. Baroreflex sensitivity, heart rate, and blood pressure variability in hypertensive pregnancy disorders. J Hum Hypertens. 2004; 18:707-712. [PubMed: 15116143]

19. Helmreich RJ, Hundley V, Varvel P. The effect of obesity on heart rate (heart period) and physiologic parameters during pregnancy. Biol Res Nurs. 2008; 10:63-78. [PubMed: 18647761]

20. Waugh J, Perry IJ, Halligan AW, et al. Birth weight and 24-hour ambulatory blood pressure in nonproteinuric hypertensive pregnancy. Am J Obstet Gynecol. 2000; 183:633-637. [PubMed: 10992185]

21. Maggioni C, Cornélissen G, Otsuka K, Halberg F, Consonni D, Nicolini U. Circadian rhythm of maternal blood pressure and fetal growth. Biomed Pharmacother. 2005; 59(Suppl 01):S86-S91. [PubMed: 16275513] 
Screened 250 subjects

Common reasons for nonenrollment

1. Incorrect gestational age

2. Did not meet inclusion criteria

3. Unreliable transportation

4. Time commitment concerns

Exclusion criteria

1) Twin pregnancy

2) First pregnancy w/o risk factors

3) Patients without obstetric care

22 Dropped:

Severe preeclampsia $(n=3)$

Other pregnancy complications $(n=1)$

Lost to follow-up $(n=12)$

Lost interest/unable to comply $(n=6)$

9 Dropped:

Severe preeclampsia $(n=2)$

Other pregnancy complications $(n=5)$

Lost interest/unable to comply $(n=2)$

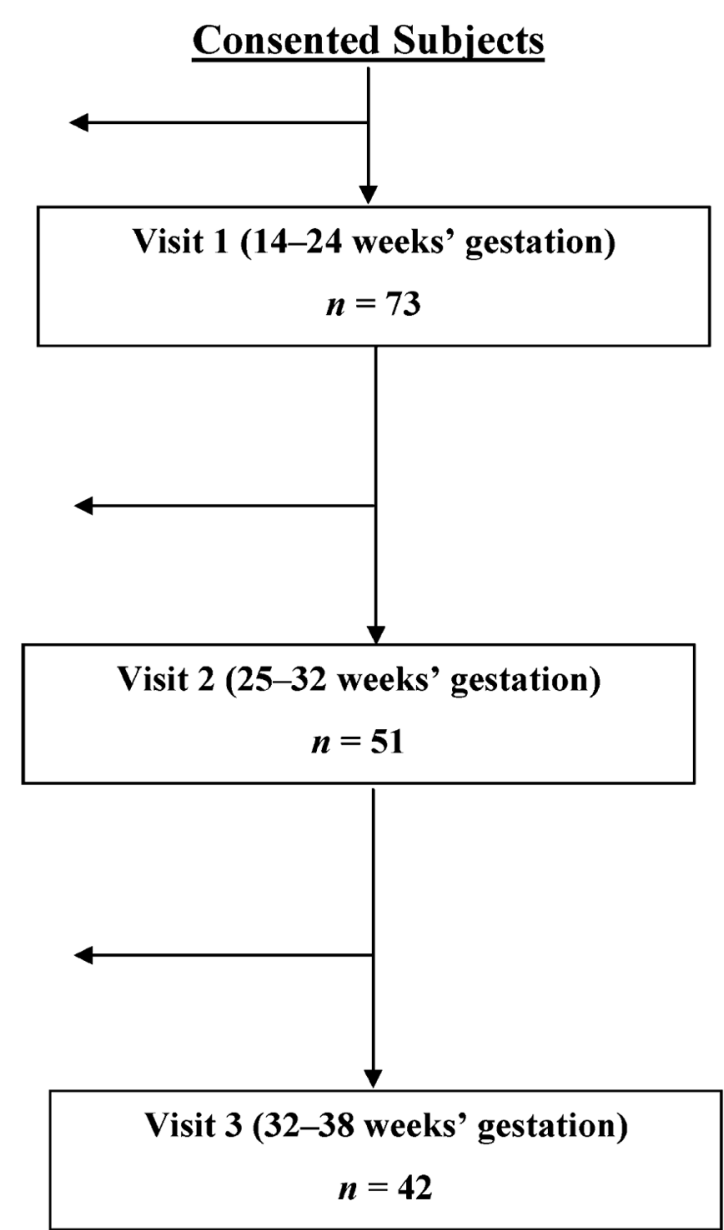

Figure 1.

Study flowchart with dropout description. 

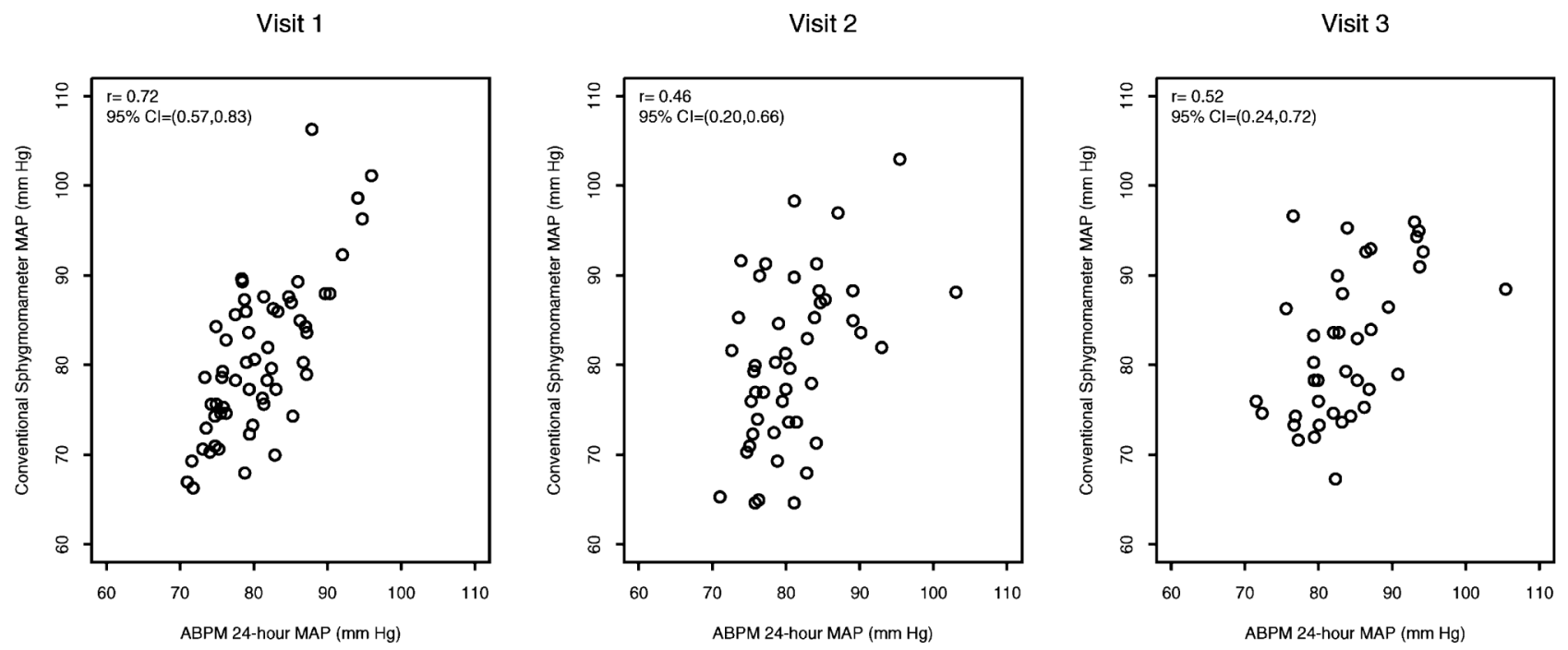

Figure 2.

Correlation between 24-hour mean arterial pressure (MAP), 24-hour ambulatory blood pressure measurements (ABPM), and conventional sphygmomanometer (CSM) blood pressure measurements. 
Visit 1

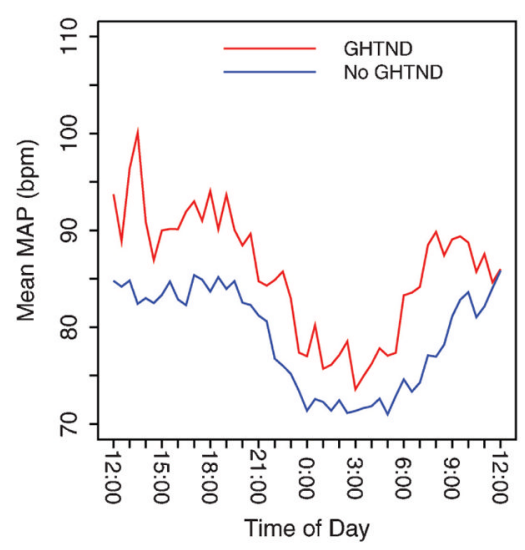

Visit 2

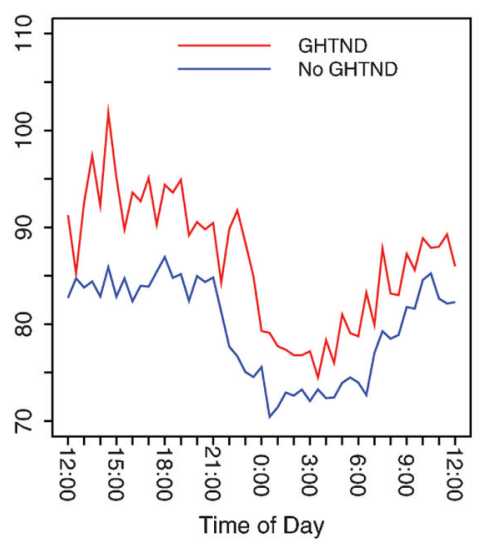

Visit 3

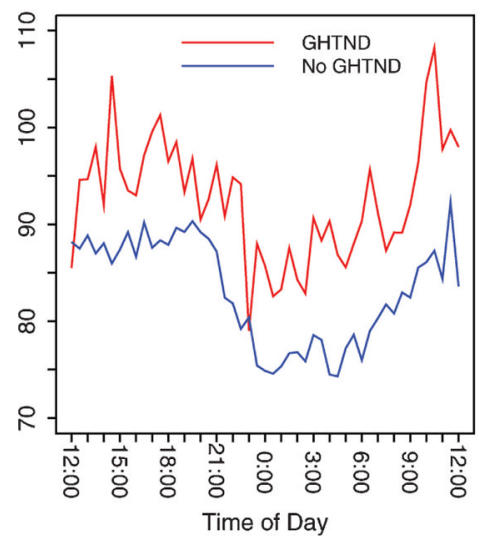

Figure 3.

Comparison of blood pressure circadian rhythms and variation in women with and without a gestational hypertension disorder (GHTND). GHTNDs include gestational hypertension, preeclampsia, and HELLP syndrome (hemolysis; elevated liver enzymes; low platelet count). Mean arterial pressure (MAP) variability increased as the pregnancy advanced in the GHTND group. There is a blunted affect in the third visit (32 weeks to delivery) in those with a GHTND. 

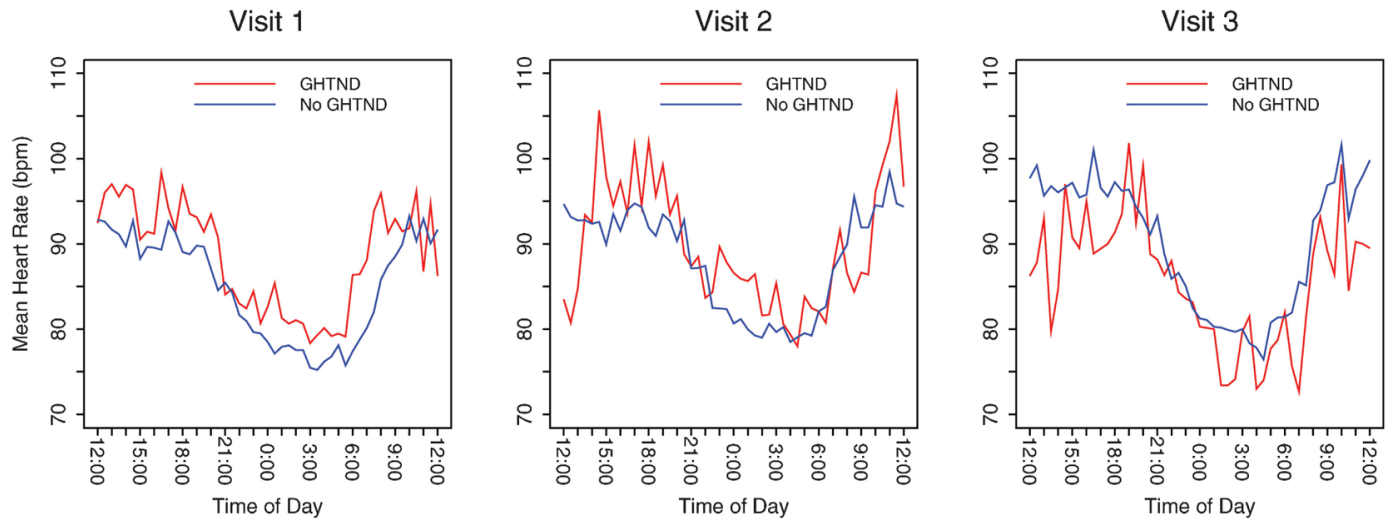

Figure 4.

Comparison of heart rate variation in women with and without gestational hypertension disorder, which includes those with gestational hypertension, preeclampsia, and HELLP syndrome (hemolysis; elevated liver enzymes; low platelet count). 
Table 1

Characteristics of Participants

\begin{tabular}{|c|c|c|c|}
\hline & $\begin{array}{l}\text { All Subjects }\left(n=7^{a}\right) \text {, Mean (SD) } \\
\text { [Range] }\end{array}$ & $\begin{array}{l}\text { GHTND }(n=17), \text { Mean }(\text { SD) } \\
\text { [Range] }\end{array}$ & $\begin{array}{l}\text { No GHTND }(n=52) \text {, Mean (SD) } \\
\text { [Range] }\end{array}$ \\
\hline \multicolumn{4}{|l|}{ Baseline demographics } \\
\hline Age (y) & $29.6(5.6)[18-41]$ & 29.5 (5.6) [19-39] & $29.4(5.6)[18-40]$ \\
\hline BMI $\left(\mathrm{kg} / \mathrm{m}^{2}\right)$ & $33.0(7.1)[15.5-57.3]$ & $34.2(6.9)[27.7-57.3]$ & $32.3(7.3)[15.5-47.7]$ \\
\hline \multicolumn{4}{|l|}{ Ethnicity/race ${ }^{\mathrm{b}}$} \\
\hline Hispanic & $7(10)$ & 0 & $7(13)$ \\
\hline Non-Hispanic white & $54(74)$ & $14(82)$ & $37(71)$ \\
\hline Non-Hispanic black & $8(11)$ & $1(6)$ & $6(12)$ \\
\hline Other & $4(5)$ & $2(12)$ & $2(4)$ \\
\hline \multicolumn{4}{|l|}{ Gravidity ${ }^{\mathrm{b}}$} \\
\hline 1 & $14(19)$ & $4(24)$ & $10(19)$ \\
\hline 2 & $22(30)$ & $3(18)$ & $18(35)$ \\
\hline 3 & $13(18)$ & $5(29)$ & $8(15)$ \\
\hline 4 & $16(22)$ & $5(29)$ & $10(19)$ \\
\hline 25 & $8(11)$ & 0 & $6(12)$ \\
\hline \multicolumn{4}{|l|}{ Parity ${ }^{b}$} \\
\hline 0 & $26(36)$ & $7(41)$ & $19(37)$ \\
\hline 1 & $23(31)$ & $4(24)$ & $18(35)$ \\
\hline 2 & $13(18)$ & $5(29)$ & $7(13)$ \\
\hline 3 & $11(15)$ & $1(6)$ & $8(15)$ \\
\hline \multicolumn{4}{|c|}{ Elective or spontaneous abortion ${ }^{\mathrm{b}}$} \\
\hline 0 & $41(56)$ & $10(59)$ & $30(58)$ \\
\hline 1 & $20(27)$ & $4(24)$ & $14(27)$ \\
\hline 2 & $6(8)$ & $2(12)$ & $4(8)$ \\
\hline$\geq 3$ & $6(8)$ & $1(6)$ & $4(8)$ \\
\hline \multicolumn{4}{|l|}{ Delivery data } \\
\hline Gestational age (wk) & $37.9(3.0)[24.1-41.6]$ & $35.8(4.4)[24.1-40.1]$ & $38.6(1.9)[31.8-41.6]$ \\
\hline Birth weight (g) & 3270 (764) [399-5235] & 2786 (1047) [399-4445] & $3428(576)[2084-5235]$ \\
\hline
\end{tabular}

BMI, body mass index; GHTND, gestational hypertension disorder; SD, standard deviation.

${ }^{a}$ Four subjects did not give authorization for the release of medical records; therefore, GHTND status was not determined. 